\title{
Visual Analytics in Environmental Decision-Making: A Comparison of Overlay Charts versus Simulation Decomposition
}

\author{
M. Kozlova ${ }^{1}$ and J. S. Yeomans ${ }^{2 *}$ \\ ${ }^{I}$ School of Business and Management, LUT University, Lappeenranta 53850, Finland \\ ${ }^{2}$ OMIS Area, Schulich School of Business, York University, 4700 Keele Street, Toronto, ON M3J 1P3, Canada \\ Received 11 May 2020; revised 30 July 2020; accepted 3 August 2020; published online 31 December 2020
}

\begin{abstract}
Various components within environmental decision-making problems often contain considerable uncertainty. Monte Carlo simulation approaches have frequently been used to incorporate a wide array of this uncertainty into environmental planning. Simulated outputs summarizing these uncertainties are commonly portrayed in the form of probability distributions. Visualization of the disparate uncertainties within these distributions is a key aspect for effective decision support in Monte Carlo analysis. This study contrasts the performance and benefits of two visual analytics tools - overlay charts and simulation decomposition. Overlay charts enable the display of multiple sources of uncertainty overlaid on top of each other in a single graphical representation and come as a standard feature in numerous commercial Monte Carlo software packages. Conversely, simulation decomposition combines user-defined sub-distributions of the simulation uncertainties and collectively displays them in a combined graphical output figure. This paper contrasts the efficacy of overlay charts versus simulation decomposition for the visual analysis uncertainty into the environmental decision-making process.
\end{abstract}

Keywords: simulation decomposition, overlay charts, Monte Carlo simulation, visual analytics, probability distribution s

\section{Introduction}

Effective environmental decision-making frequently proves to be a complex, challenging process (Loughlin et al., 2001; Janssen et al., 2010) in which the final solutions often feature political, socio-economic, and environmental aspects that are inherently subjective (Loughlin et al., 2001; Zechman and Ranjithan, 2004). Although certain components may be articulated clearly, typical environmental decision-making problems possess modelling specifications that cannot be directly incorporated into the underlying decision support process (Hipel and Ben-Haim, 1999; Mowrer, 2000; De Kok and Wind, 2003; Brugnach et al., 2007; Matthies et al., 2007; Fuerst et al., 2010; Hipel and Walker, 2011; Castelletti et al., 2012; Lund, 2012; Walker et al., 2012; Deviatkin et al., 2020). Environmental decision-making can be further compounded in circumtances where stochastic uncertainties are prevalent (Baetz, 1990; Yeomans, 2008; Gunalay et al., 2012; Farr et al., 2016; Han et al., 2017; Kozlova and Yeomans, 2019).

Monte Carlo methods have been integrated into a variety of environmental contexts to incorporate these uncertainty issues (see, for example: Openshaw and Whitehead, 1985; Ridlehoover, 2004; Byer and Yeomans, 2007; Byer et al., 2009; Byer et al., 2011; Vithayasrichareon and Macgill, 2012; Kim et al.,

${ }^{*}$ Corresponding author. Tel.: 416-736-5074.

E-mail address: syeomans@schulich.yorku.ca (J. S. Yeomans).

ISSN: 2663-6859 print/2663-6867 online

(C) 2020 ISEIS All rights reserved. doi:10.3808/jeil.202000047.
2013; Farr et al., 2016; Han et al., 2017). An effective simulation analysis should capture not only the ranges of realistic possible outcomes, but also the distributional nature of how the identified risks actually "behave" between identified extremes (Byer et al., 2011; Kleijnen, 2018; Kozlova and Yeomans, 2019). Monte Carlo simulation can convey the potential impacts on different systems based upon the likelihoods of certain events (Byer and Yeomans, 2007; Byer et al., 2009). Since the resulting outputs are best articulated graphically as probability distributions, simulation output generally displays a more nuanced, visual portrayal of system performance than that of a single, deterministic value (Kleijnen, 2018).

While simulation integrates stochastic behaviours into the modelling, it provides no prescriptive mechanism that actually creates system solutions. Hence, all key conclusions revert to the decision-makers studying the uncertainties embodied by the distributional outputs (Byer et al., 2009). Although Monte Carlo simulation has been applied to wide spectrum of problems (Law and Kelton, 2000), the approach to its output analysis has remained comparatively static (Kleijnen, 2018). Typically, simulation-created output distributions are portrayed in the form of histograms and, therefore, visualization becomes an important decision-making component when the models contain multiple sources of uncertain variable interaction. Visual analytics is the graphic representation of data to analyze, process, uncover, and communicate relationships among the represented data.

Perhaps the most prevalent visualization tool available in commercial Monte Carlo software packages is the overlay chart. 
Overlay charts enable the simultaneous viewing, comparison, and display of several stochastically uncertain components all concurrently displayed on the same chart (Palisade, 2015; Oracle, 2020). After completing a simulation with multiple related forecasts, an overlay chart is created to display the relative characteristics of those forecasts on a single chart. The frequency data from selected forecasts is superimposed in one chart to highlight similarities and differences that otherwise might not be readily apparent. There is no limit to the number of forecasts you can view on an overlay chart at one time. Namely, the overlay chart displays multiple sets of data placed one on top of the other. Displaying the data in this fashion makes it easier to visually identify events that coincide with one another and to quickly determine if a correlation or relationship exists between them. While correlation obviously does not imply causation, theorizing based upon the data displayed in an overlay chart can justify further investigation by the decision-makers to identify the root causes of an issue.

Recently, a new visualization tool referred to as simulation decomposition ( $\mathrm{SimDec}$ ) has been introduced that extends Monte Carlo analysis by enhancing the explanatory power of the cause-effect relationships between the multi-variable combinations of inputs and the simulated outputs. The universality of SimDec and its usefulness in planning across many different disciplines has been outlined in Kozlova and Yeomans (2020). SimDec partitions sub-distributions of the Monte Carlo outputs by pre-classifying selected input variables into states, grouping combinations of these states into scenarios, and then collecting simulated outputs attributable to each multi-variable input scenario. Since it is a straightforward task to visually project the contribution of these subdivided scenarios onto the overall output chart, SimDec enables a visualization of previously unidentified connections between multi-variable combinations of inputs on the outputs. SimDec is generalizable to any simulation method without the need for any significant additional computing resources. SimDec's innovative visual analytics capabilities have already been considered in a diverse range of environmental decision-making problems (Kozlova et al., 2016; Kozlova and Yeomans, 2019; Deviatkin et al., 2020; Hietanen, 2020; Sadyhova, 2020). Although not commercially available, downloadable versions of SimDec code are readily accessible in Matlab (Kozlova et al., 2018a, 2018b), VBA for Excel (Kozlova and Yeomans, 2020), Python, and R (Sadyhova, 2020).

Visualization of the disparate uncertainties within Monte Carlo output distributions is a key aspect for effective decision support in environmental decision-making. This study specifically contrasts the performance and benefits of two significant, visual analytics tools - overlay charts and simulation decomposition. Overlay charts enable the display of multiple sources of uncertainty stacked on top of each other in a single graphical representation and come as a standard feature in numerous commercial Monte Carlo software packages. Conversely, simulation decomposition combines user-defined sub-distributions of the simulation uncertainties and collectively displays them in a combined graphical output figure. Consequently, this paper aims to compare and contrast the efficacy of overlay charts versus simulation decomposition for visually analyzing uncertain- ty in the environmental decision-making process.

\section{Methods Overview}

This section provides a synopsis of the Monte Carlo simulation technique followed by a brief overview of overlay charts and SimDec.

\subsection{Monte Carlo Simulation}

Monte Carlo simulation is a straightforward method for assessing the sensitivity of the outputs of a modelled system to the uncertainty of its input factors. To perform a Monte Carlo simulation, one should: (i) estimate the uncertainty of input variables, their ranges, and/or their distributions; (ii) run the model multiple times altering the input variable values randomly according to the defined distributions; and, (iii) record the output variable values for each model run. The resulting vector of output variables can be analyzed statistically (e.g., by calculating minimum, maximum, means, and variance) and/or be displayed graphically as a probability distribution (e.g., a histogram). Graphical depictions of simulated distributions provide an effecttive visual perception of the uncertainty inherent in the outcome variables - especially their overall range, shape, and relative tendencies.

\subsection{Overlay Charts}

As mentioned in the introduction, overlay charts are amongst the most predominant visualization tools currently available in commercial Monte Carlo software packages (Mun 2010; Palisade 2015; Oracle 2020;). In overlay charts, input distributions are truncated to reflect specific decision-maker assumptions and separate simulations are run for each truncated set of inputs. Each of these separate distributions is then projected onto a single chart, with one distribution laid (or overlaid) on top of another. The approach has frequently been employed for visual analysis in risk analysis (Mun, 2010). Overlay charts are an implementation of a simple idea to enable the display of numerous stochastically uncertain outcomes from several different Monte Carlo simulations concurrently in a single graph. Figure 1 illustrates two overlay charts generated by Palisade's @RISK (Palisade, 2015) and Oracle ${ }^{\circledR}$ Crystal Ball (Oracle, 2020).

\subsection{Simulation Decomposition}

In contrast to overlay charts, SimDec permits the simultaneous display of numerous stochastically uncertain outcomes from a single Monte Carlo simulation run. SimDec constructs sub-distributions of the simulation output by pre-classifying some of the uncertain input variables into states, clustering the various combinations of these different states into scenarios, and then collecting simulated outputs attributable to each multivariable input scenario (Kozlova and Yeomans, 2019). Since the contribution of subdivided scenarios to the overall output is easily portrayed visually, SimDec can highlight and disclose previously unidentified connections between the multi-variable combinations of inputs on the outputs. This, in turn, enables decom- 
posing the probability distribution into the outcomes of different scenarios. Notably, this approach preserves the shape of the original probability distribution obtained from a classical Monte Carlo simulation, and simply color-codes different areas of this distribution following scenario matching. A SimDec approach is generalizable to any Monte Carlo model with negligible additional computational overhead and, hence, can be readily used for environmental analyses that employ simulation models. A comprehensive method description and the implementation procedure can be found in Kozlova and Yeomans, 2019.
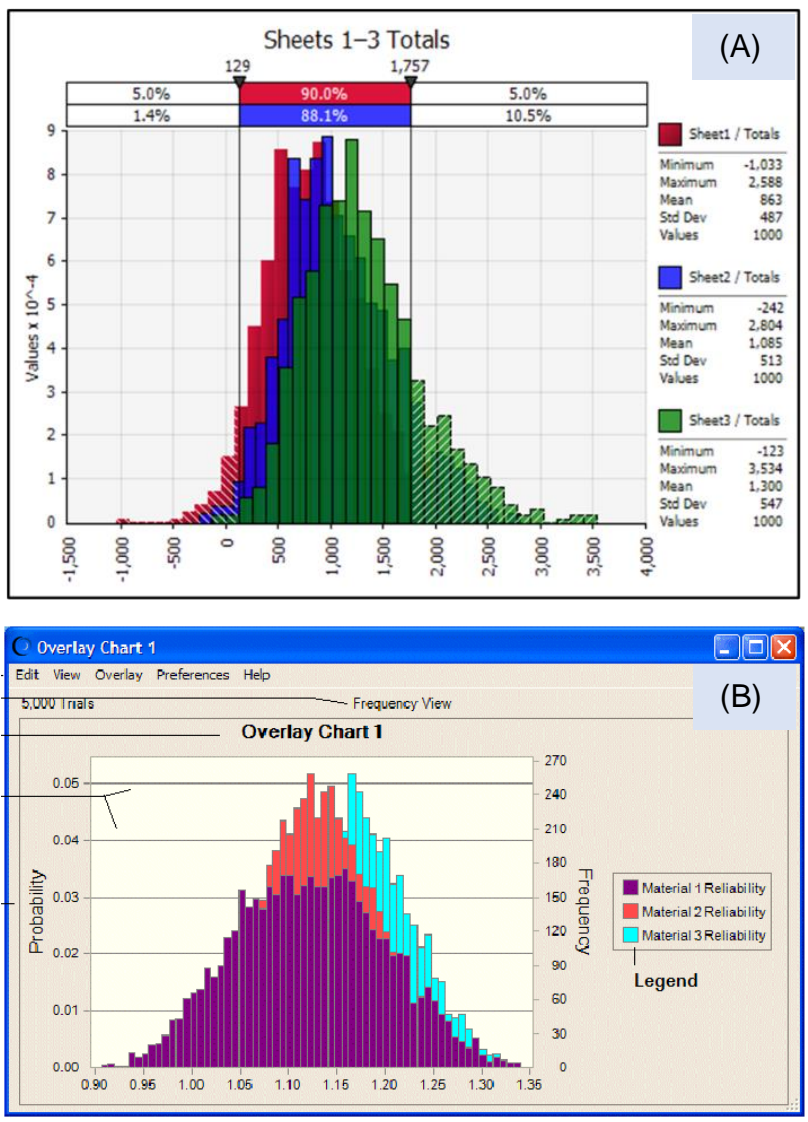

Figure 1. Overlay charts by (A) Palisade's @RISK (Palisade, 2015) and (B) Oracle $®$ Crystal Ball (Oracle, 2020).

\section{A Comparison of Overlay Charts versus Simulation Decomposition}

In order to compare the basic features of overlay charts and SimDec, consider the following oversimplified mathematical model:

$Y=A \times k+B$

where $Y$ is the single output variable of interest; $A, B$, and $k$ are all input variables. For simplicity, $A$ and $B$ are both assumed to be uniformly distributed in the range $[0,100]$ and $k=2$. Consequently, values of the output variable will be distributed over the range $[0,300]$. The scenarios for decomposition and over- lay charts are determined based upon a partitioning of variable $A$ into the ranges [0, 20], $(20,80]$, and $(80,100]$. A Monte Carlo simulation of the model was then run 10,000 times. Figure 2 depicts the various graphical representations of $Y$ produced by: (A) the overall Monte Carlo simulation; (B) SimDec; and, (C) overlay charts.
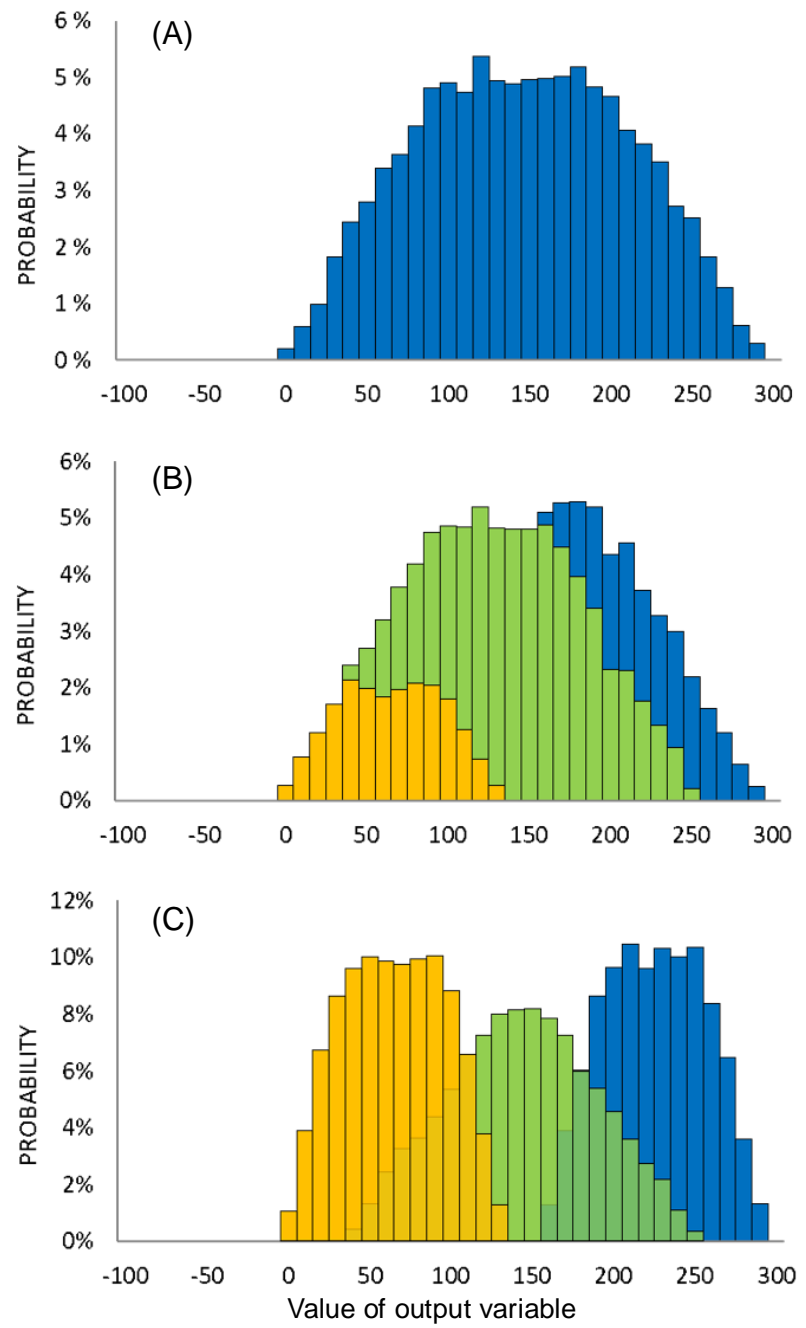

Figure 2. A simple abstract model represented by (A) Monte Carlo simulation, (B) SimDec, and (C) Overlay charts with a low number of slightly-overlapping scenarios.

Based upon initial visual impressions, the overlay chart in Figure $2 \mathrm{C}$ appears to provide the clearest visual breakdown by scenario of the output uncertainty. However, the overlay chart does not effectively capture the inherent asymmetry of scenario probabilities, so the visual representation is relatively misleading. Namely, the cumulative probability of each scenario in the overlay chart is 1 , because each scenario is generated from a separate simulation. Due to different distribution widths, Scenarios 1 and 3 appear relatively larger (or more probable) than Scenario 2 in the middle, although the converse is, in fact, the case.

In SimDec (Figure 2B), the relative areas of each scenario to the others corresponds proportionately, in size, to the relation 
of their relative probabilities. Indeed, the green area (Scenario 2 ) in Figure 2B appears substantially larger than either of the yellow (Scenario 1) and blue (Scenario 3) areas. This relative sizing corresponds to a probability of 0.6 for Scenario 2 in contrast to a probability of 0.2 for each of Scenarios 1 and 3 . Thus, when a decision-maker observes the stacked visual representation of SimDec, the relative probabilities of scenarios can be perceived intuitively based upon the relative area sizes of each scenario region.

(A)

\begin{tabular}{|c|c|c|c|}
\hline Color & Scenario & A & B \\
\hline 。 & 1 & \multirow{3}{*}{ low } & low \\
\hline \multirow[t]{2}{*}{$\circ$} & 2 & & medium \\
\hline & 3 & & high \\
\hline$\circ$ & 4 & \multirow{3}{*}{ medium } & low \\
\hline$\circ$ & 5 & & medium \\
\hline- & 6 & & high \\
\hline \multirow[t]{2}{*}{$\circ$} & 7 & \multirow{3}{*}{ high } & low \\
\hline & 8 & & medium \\
\hline$\circ$ & 9 & & high \\
\hline
\end{tabular}
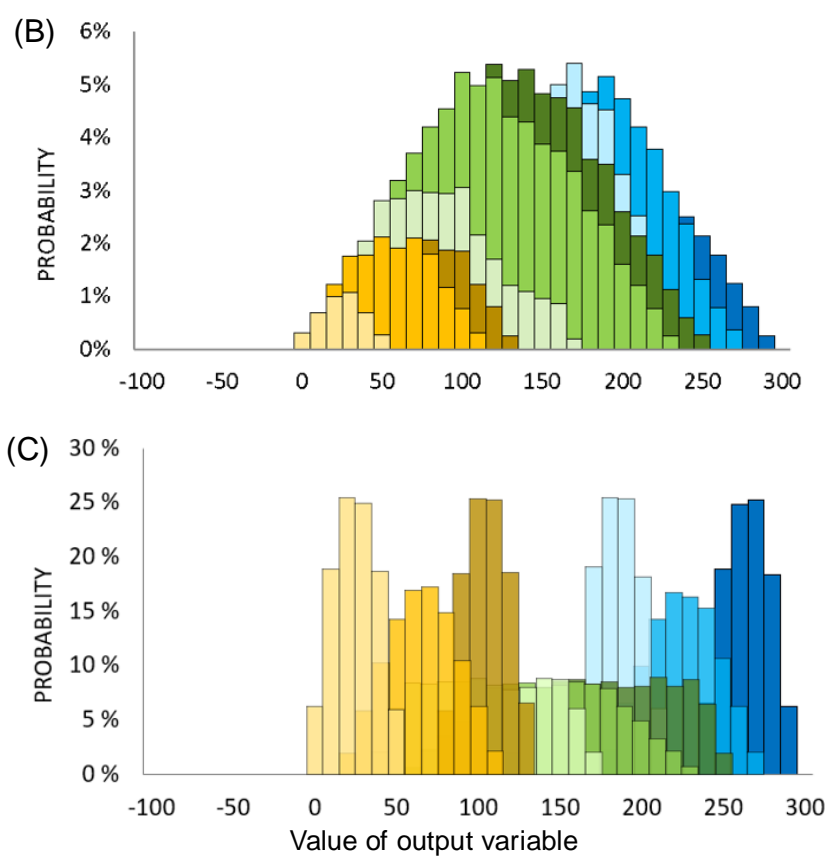

Figure 3. The same model represented by (B) SimDec and (C) Overlay charts with a high number of overlapping scenarios with the (A) legend for both graphs.

The asymmetrical issues observed in overlay charts can be circumvented only if the scenario ranges have been established congruently. This situation is rarely the case, since meaningful scenarios reflecting project realities do not generally consist of equally sized states for all of the input variable ranges. Under such circumstances, visualization in overlay charts can only be accomplished if an additional 'full' simulation has been performed that establishes the relative occurrence probabilities for each scenario and the size of each scenario's histogram is then subsequently adjusted proportionately by this probability. Such adjustments require significant additional coding which proves challenging to implement using existing commercial software.

Furthermore, any initial, yet illusory, visual clarity attributable to overlay charts rapidly dissipates with increasing numbers of scenario combinations. For example, with the three extra states, $[0,20],(20,80]$, and $(80,100]$, added for variable $B$, the original problem will now be comprised of the nine scenarios listed in the legend of Figure 3A.

With nine scenarios, the overlay chart (Figure 3C) now conceals significant portions of critical information and markedly distorts the relative scenario occurrence probabilities. Scenario 5 appears as one of the smallest in Figure 3C, while, in reality, it actually occurs most frequently. Furthermore, the boundaries of the distributions are not visible even with a partially transparent shading of the bars. More significantly, overlay charts are unable to preserve the shape of overall output distributions because they fail to account for the relative probabilities of the sub-distributions contained within them. Using overlay charts can be complicated because (i) one either needs commercial software to create it or (ii) one must run the same simulation model multiple times while changing inputs, saving multiple model versions, and concatenating the graphs together manually at the conclusion. The latter procedure is computationally intensive if performed on one set of scenarios and becomes prohibitively expensive time-wise if repeated several times to account for different sets of scenarios in an exploratory analysis. Finally, when a problem is approached using overlay charts from the outset, scenario setting becomes an arbitrary task and can fail to consider potentially critical combinations of variable states.

Conversely, SimDec (Figure 3B) clearly displays that the lower boundary of Scenario 7 is considerably higher than the lower boundary of Scenario 6. It would be impossible to draw such an inference from the overlay chart. One disadvantage of SimDec is that it has the potential to distort the relative shapes of some sub-distributions. Since some sub-distributions are stacked on top of each other, the shape of one sub-distribution inherently depends on the shapes of any sub-distributions below it. As noted in the introduction, SimDec software is freely available in several computer languages including an Excel version and can be easily implemented as an add-on to any existing Monte Carlo model. A side-by-side comparison of the pros and cons of overlay charts versus SimDec is presented in the Table 1.

Recently, the visual analytics capabilities of SimDec have been used to produce novel insights into such diverse environmental decision-making problems as renewable energy policy analysis (Kozlova et al., 2016; Hietanen, 2020), environmental policy planning (Kozlova and Yeomans, 2019), carbon footprint analysis (Deviatkin et al., 2020), and green construction (Sadyhova, 2020). Hietanen (2020) demonstrated how performing a decomposition of renewable energy policy with different sets of factors can uncover additional insights and provide a comprehensive understanding of the problem's underlying complexities. Figure 4 provides a summary of SimDec's visualization output from these various complex, multifaceted environmental decision-making problems (Kozlova et al., 2016; Kozlova and Yeomans, 2019; Deviatkin et al., 2020; Hietanen, 2020). 
Since one cannot combine separate overlay components into a single output distribution chart unless their likelihoods have been weighted by their proportional occurrences (which cannot be determined without running a complete simulation over all the ranges simultaneously), it would seem logical to deduce that overlay charts would not have been able to approach these problems as comprehensively as SimDec.

\section{Conclusions}

Environmental decision-making frequently requires the need for the practical, "real world" analysis of applications possessing numerous uncertain factors and unquantified dimensions. Monte Carlo simulation has been applied to a wide array of environmental planning settings to incorporate these uncertain features and the outputs are commonly displayed as probability distributions. Data visualization and visual analytics provide decision support tools for the processing, analyzing, and communicating of these uncertainties in the decision-making proprocess. This study has contrasted the efficacy of overlay charts and simulation decomposition in visually analyzing uncertainty in Monte Carlo-based environmental decision-making processes.

Table 1. Features, Pros, and Cons of SimDec and Overlay Charts

\begin{tabular}{|c|c|c|}
\hline Feature & SimDec & Overlay charts \\
\hline Histogram type & Stacked & Overlaid \\
\hline Simulation runs & Once & Equal to the number of scenarios \\
\hline $\begin{array}{l}\text { Visibility of edges of the } \\
\text { scenarios }\end{array}$ & Edges of each sub-distribution are clearly visible & $\begin{array}{l}\text { Edges of some distributions are hidden. The more } \\
\text { scenarios and the bigger their overlap, the poorer } \\
\text { visibility. Making chart 3D or the histogram half- } \\
\text { transparent has only a limited positive effect }\end{array}$ \\
\hline Inter-location of scenarios & $\begin{array}{l}\text { Inter-location of any pair of scenarios can be easily } \\
\text { analyzed since all the sub-distributions are entirely } \\
\text { visible }\end{array}$ & $\begin{array}{l}\text { Inter-location analysis is hindered due to visibility } \\
\text { issues }\end{array}$ \\
\hline Shape of sub-distributions & $\begin{array}{l}\text { The shape of sub-distributions is challenging to read } \\
\text { because sub-distributions are stacked into one chart, and } \\
\text { the shape of one would depend on the shape of all others } \\
\text { beneath it }\end{array}$ & $\begin{array}{l}\text { The shape of distributions is preserved but might } \\
\text { be partially hidden }\end{array}$ \\
\hline $\begin{array}{l}\text { The shape of the overall } \\
\text { distribution }\end{array}$ & $\begin{array}{l}\text { The shape of the overall distribution (if as only classical } \\
\text { Monte Carlo simulation is run) is preserved. The sub- } \\
\text { distributions are displayed as a portion of the whole }\end{array}$ & $\begin{array}{l}\text { The shape of the overall distribution is not } \\
\text { preserved since, for every distribution, the } \\
\text { simulation is run again }\end{array}$ \\
\hline Universal set of scenarios & $\begin{array}{l}\text { SimDec, by default, decomposes the entire universal set, } \\
\text { e.g., each variable has its defined minimum and } \\
\text { maximum and all possible combinations of those in the } \\
\text { outcome }\end{array}$ & $\begin{array}{l}\text { The combined set of outcomes depends on the } \\
\text { definition of scenarios and might not necessarily } \\
\text { constitute the universal set, so some potentially } \\
\text { important uncertainty zones might be missed out }\end{array}$ \\
\hline $\begin{array}{l}\text { Asymmetric frequency of } \\
\text { scenarios }\end{array}$ & $\begin{array}{l}\text { Able to portray asymmetric probability of different } \\
\text { scenario outcomes based on the combinations of } \\
\text { predefined states and original distributions of input } \\
\text { variables since each sub-distribution is a product of a } \\
\text { single Monte Carlo simulation. The cumulative } \\
\text { probability of the sum of all scenarios is } 100 \%\end{array}$ & $\begin{array}{l}\text { Not able to capture asymmetric probability of } \\
\text { different scenarios. Because a new simulation is } \\
\text { run for each scenario, the cumulative probability } \\
\text { of each scenario is } 100 \%\end{array}$ \\
\hline Number of scenarios & $\begin{array}{l}\text { Able to display as many scenarios as required. The color- } \\
\text { coding becomes important for facilitating visual } \\
\text { perception (e.g., the most influential variable with } \\
\text { different colors, less influential with shades of those } \\
\text { colors) }\end{array}$ & $\begin{array}{l}\text { With a higher number of scenarios, the visual } \\
\text { perception deteriorates, and more information is } \\
\text { hidden }\end{array}$ \\
\hline Computational costs & $252 \mathrm{sec}$ with Ms. Excel for nine scenarios & $\begin{array}{l}1539 \mathrm{sec} \text { with Ms. Excel running nine separate } \\
\text { simulations }\end{array}$ \\
\hline Implementation & $\begin{array}{l}\text { Adding a module recording the values of the key input } \\
\text { variables during the simulation, scenario matching \& } \\
\text { histogram color-coding }\end{array}$ & $\begin{array}{l}\text { (i) The original Monte Carlo simulation model can } \\
\text { be used without changes, but the approach will } \\
\text { require running it multiple times and manually } \\
\text { overlaying the charts } \\
\text { (ii) An alternative is to create a code that runs the } \\
\text { simulation multiple times and overlays the chart } \\
\text { automatically }\end{array}$ \\
\hline Availability & $\begin{array}{l}\text { SimDec is realized in multiple computer languages and } \\
\text { available openly for use }\end{array}$ & $\begin{array}{l}\text { (i) It can be implemented by the user based on the } \\
\text { existing Monte Carlo model } \\
\text { (ii) Commercial software }\end{array}$ \\
\hline Operation & $\begin{array}{l}\text { No changes to the inputs are required, the model is saved } \\
\text { once, and it contains all decomposition information }\end{array}$ & $\begin{array}{l}\text { (i) It requires changing the inputs for every } \\
\text { scenario and either saving multiple models or } \\
\text { losing the data } \\
\text { (ii) Ok, but not free }\end{array}$ \\
\hline
\end{tabular}



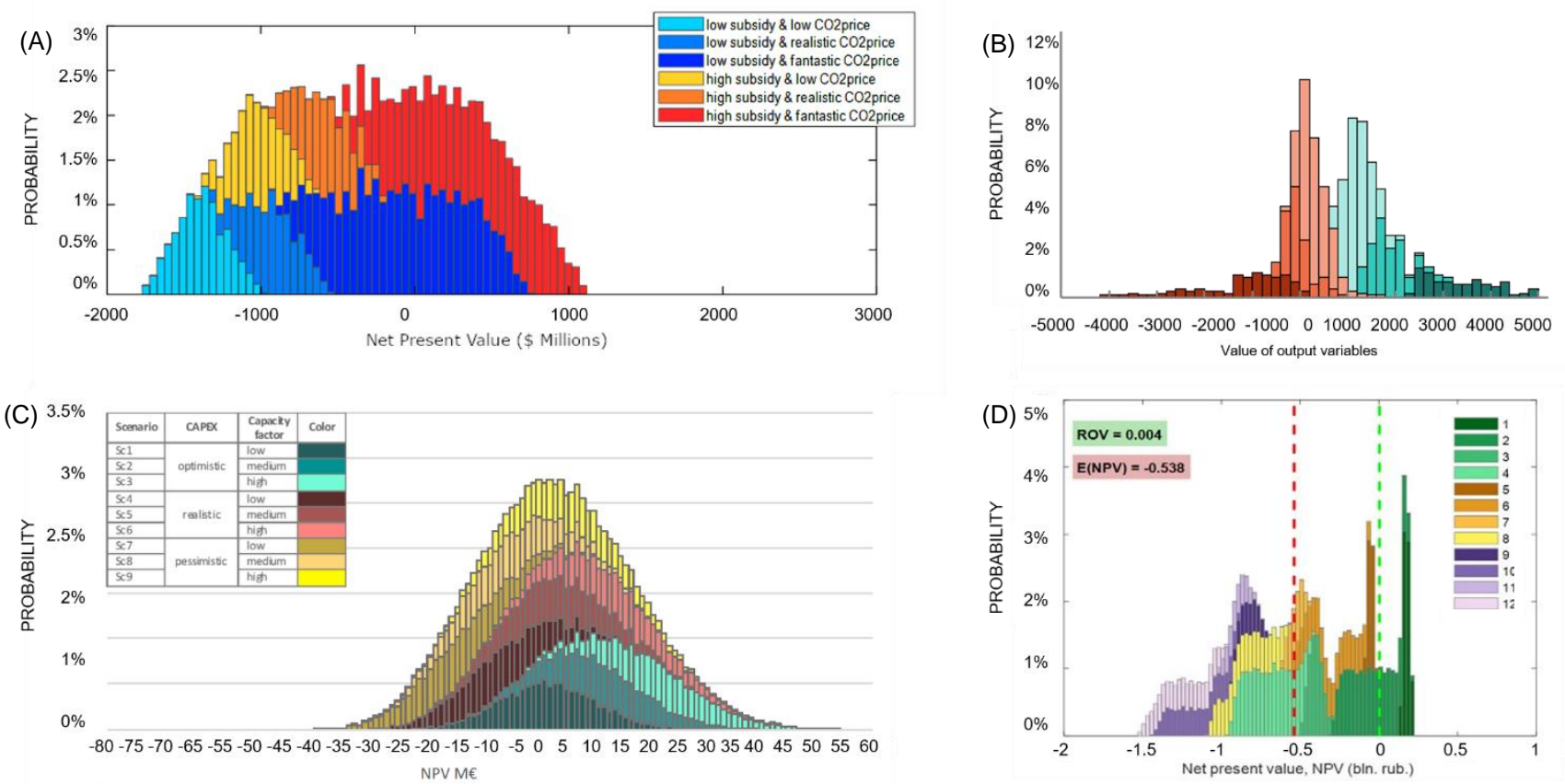

Figure 4. SimDec usage in the academic literature: (A) value of a CCS investment for a coal plant in china under carbon trading

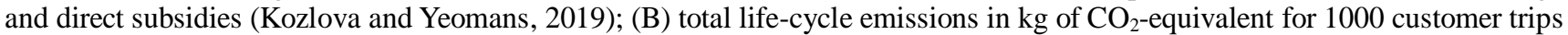
with wooden pallets (Deviatkin et al., 2020); (C) wind power project NPV under finnish feed-in tariffs (Hietanen, 2020); (D) NPV of a wind farm investment under russian renewable energy support (Kozlova et al., 2016).

Overlay charts represent one of the most predominant visualization tools available in comercial Monte Carlo software packages for simultaneously comparing several stochastically uncertain components. SimDec is a recently introduced visual analytics tool for evaluating potential cause-effect relationships of multi-variable combinations of inputs on the corresponding outputs in Monte Carlo simulation. While overlay charts display multiple individual sets of data one-on-top-of-another, SimDec permits the simultaneous projection of several combined multivariable stochastic uncertainties directly onto a single output distribution.

SimDec provides the ability to observe the total output distribution of variables of interest combined with the simultaneous ability to visually detect which components of this overall distribution are attributable to specific sets of combinations of input variables. These input variable combinations are userspecified and can be changed in an exploratory fashion to reflect other combinations of input variables. Hence, a decomposition provides an immediate visual perception of the impacts on the output distribution of interest. This decomposed total visualization of the output represents what most decision-makers wish to observe. Significantly, an analogous visual breakdown cannot be provided by the overlay chart option without considerable additional computational effort.

Since SimDec is completely generalizable to any Monte Carlo application with only negligible additional computational overhead, it can be readily extended into many environmental decision-making instances. In addition, decision-makers can visually perform exploratory analysis of the outputs by construc- ting a variety of different multi-variable input combination scenarios and examining the resulting graphical projections onto the output distribution figures. This paper has demonstrated that SimDec's complete generalizability combined with its straightforward visualizations of complex stochastic uncertainties render the technique so inherently practical and, perhaps, significantly more adaptable than overlay charts for Monte Carlo-based, environmental decision-making applications.

Acknowledgement. This research was supported in part by funding from the Finnish Strategic Research Council, grant number 313396/ MFG40 - Manufacturing 4.0, by grant number 190197 from The Foundation for Economic Education, Finland, and by grant OGP0155871 from the Natural Sciences and Engineering Research Council.

\section{References}

Baetz, B.W. (1990). Optimization/simulation modeling for waste management capacity planning. ASCE Journal of Urban Planning and Development, 116 (2), 59-79. https://doi.org/10.1061/(ASCE) 07339488(1990)116:2(59)

Brugnach, M., Tagg, A., Keil, F., and De Lange, W.J. (2007). Uncertainty matters: computer models at the science-policy interface. Water Resources Management, 21, 1075-1090. https://doi.org/10. 1007/s11269-006-9099-y

Byer, P.H. and Yeomans, J.S. (2007). Methods for addressing climate change uncertainties in project environmental impact assessments. Impact Assessment and Project Appraisal, 25(2), 85-99. https:// doi.org/10.3152/146155107X205841

Byer, P.H., Lalani, M.J., and Yeomans, J.S. (2009). Addressing and communicating climate change and its uncertainties in project envi- 
ronmental impact assessments. Journal of Environmental Assessment Policy and Management, 11(1), 29-50. https://doi.org/10.11 42/S1464333209003245

Byer, P.H., Colombo, A.F., Sabelli, A., and Ches, C. (2011). Decision making under uncertainties for adapting to climate change in project environmental assessments. Research report to the Canadian Environmental Assessment Agency (CEAA).

Castelletti, A., Galelli, S., Restelli, M., and Soncini-Sessa, R. (2012). Data-driven dynamic emulation modelling for the optimal management of environmental systems. Environmental Modelling \& Software, 34(3), 30-43. https://doi.org/10.1016/j.envsoft.2011. 09.003

De Kok, J.L. and Wind, H.G. (2003). Design and application of decision support systems for integrated water management; lessons to be learnt. Physics and Chemistry of the Earth, 28(14-15), 571-578. https://doi.org/10.1016/S1474-7065(03)00103-7

Deviatkin, I., Kozlova, M., and Yeomans, J.S. (2020). Simulation decomposition for environmental sustainability: Enhanced decisionmaking in carbon footprint analysis. Socio-Economic Planning Sciences. https://doi.org/10.1016/j.seps.2020.100837

Fan, J., Xu, M., Wei, S., Zhong, P., Zhang, X., and Yang, Y. (2018). Evaluating the effect of a subsidy policy on carbon capture and storage (ccs) investment decision-making in china - a perspective based on the 45q tax credit. Energy Procedia, 154, 22-28. https://doi. org/10.1016/j.egypro.2018.11.005

Farr, J.V., Faber, I.J., Ganguly, A., Martin, W.A., and Larson, S.L. (2016). Simulation-based costing for early phase life cycle cost analysis: example application to an environmental remediation project. Engineering Economist, 61(3), 207-222. https://doi.org/10.1080/ 0013791X.2015.1062582

Fuerst, C., Volk, M., and Makeschin, F. (2010). Squaring the circle? Combining models, indicators, experts and end-users in integrated land-use management support tools. Environmental Management, 46(6), 829-833. https://doi.org/10.1007/s00267-010-9574-3

Gunalay, Y., Yeomans, J.S., and Huang, G. (2012). Modelling to generate alternative policies in highly uncertain environments: An application to municipal solid waste management planning. Journal of Environmental Informatics, 19(2), 58-69. https://doi.org/10.3808/ jei.201200209

Han, Z., Porras-Alvarado, J.D., and Sun, J. (2017). Monte Carlo simulation-based assessment of risks associated with public-private partnership investments in toll highway infrastructure. Journal of the Transportation Research Board, 2670(1), 59-67. https://doi.org/10. $3141 / 2670-08$

Hietanen, L. (2020). Comparative analysis of renewable energy policy schemes of Finland. Master's thesis, LUT University, Lappeenranta, Finland. http://urn.fi/URN:NBN:fi-fe2020062545726

Hipel, K. and Ben-Haim, Y. (1999). Decision making in an uncertain world: information-gap modeling in water resources management. IEEE Transactions on Systems, Man and Cybernetics - part C: Applications and Reviews, 29(4), 506-517. https://doi.org/10.1109/ 5326.798765

Hipel, K.W. and Walker, S.G.B. (2011). Conflict analysis in environmental management. Environmetrics, 22(3), 279-293. https://doi. org/10.1002/env. 1048

Janssen, J.A.E.B., Krol, M.S., Schielen, R.M.J. and Hoekstra, A.Y. (2010). The effect of modelling quantified expert knowledge and uncertainty information on model-based decision making. Environmental Science and Policy, 13(3), 229-238. https://doi.org/10.1016/j. envsci.2010.03.003

Kim, T.H., Choi, J.S, Park, Y.J., and Son, K. (2013). Life cycle costing: Maintenance and repair costs of hospital facilities using Monte Carlo simulation. The Korean Institute of Building Construction, 13(6), 541-548. https://doi.org/10.5345/JKIBC.2013. 13.6.541

Kleijnen, J.P.C. (2018). Design and analysis of simulation experiments. In: Pilz J., Rasch D., Melas V., Moder K. (eds) Statistics and Simulation. IWS 2015. Springer Proceedings in Mathematics \& Statistics.
Cham, Switzerland. 231, 3-22. https://doi.org/10.1007/978-3-31976035-3_1

Kozlova, M. and Yeomans, J.S. (2019). Multi-variable simulation decomposition in environmental planning: An application to carbon capture and storage. Journal of Environmental Informatics Letters, 1(1), 20-26. https://doi:10.3808/jeil.201900003

Kozlova, M. and Yeomans, J.S. (2020). Monte Carlo enhancement with simulation decomposition: A "must-have" for many disciplines. INFORMS Transactions on Education.

Kozlova, M., Collan, M., and Luukka, P. (2016). Simulation decomposition: New approach for better simulation analysis of multi-variable investment projects. Fuzzy Economic Review, 21(2), 3. https:// doi.org/10.25102/fer.2016.02.01

Kozlova, M., Collan, M. and Luukka, P. (2018a). Simple example for decomposition. Retrieved August 6, 2019, from https://www.resear chgate.net/publication/322211248_simple_example_for_decompos ition

Kozlova, M., Collan, M. and Luukka, P. (2018b). Matlab function for simulation decomposition. Retrieved August 6, 2019, from https:// www.researchgate.net/publication/322211201_matlab_function_fo r_simulation_decomposition

Law, A.M. and Kelton W.D. (2000). Simulation Modeling and Analysis (3rd eds). New York: McGraw-Hill.

Loughlin, D.H., Ranjithan, S.R., Brill E.D. and Baugh, J.W. (2001). Genetic algorithm approaches for addressing unmodeled objectives in optimization problems. Engineering Optimization, 33(5), 549569. https://doi.org/10.1080/03052150108940933

Lund, J. (2012). Provoking more productive discussion of wicked problems. Journal of Water Resources Planning and Management, 138(3), 193-195. 195. https://doi.org/10.1061/(ASCE)WR.1943-54 52.0000190

Matthies, M., Giupponi, C., and Ostendorf, B. (2007). Environmental decision support systems: Current issues, methods and tools. Environmental Modelling \& Software, 22(2), 123-127. 127. https://doi. org/10.1016/j.envsoft.2005.09.005

Mowrer, H.T. (2000). Uncertainty in natural resource decision support systems: Sources, interpretation and importance. Computers and Electronics in Agriculture, 27(1-3), 139-154. 154. https://doi.org/10. 1016/S0168-1699(00)00113-7

Mun, J. (2010). Modeling risk: Applying Monte Carlo risk simulation, strategic real options, stochastic forecasting, and portfolio optimization. John Wiley and Sons.

Openshaw, B.W. and Whitehead, P. (1985). A Monte Carlo simulation approach to solving multicriteria optimization problems related to plan making, evaluation, and monitoring in local planning. Environment and Planning B: Planning and Design, 12, 321-334. 334. https://doi.org/10.1068/b120321

Oracle. (2020). Oracle ${ }^{\circledR}$ Crystal Ball user's guide: Creating overlay charts. Retrieved June 16, 2020. https://docs.oracle.com/cd/E5718 5_01/CYBUG/overlay_charts_dialog.htm

Palisade. (2015). Palisade knowledge base: @RISK Automatic overlays from multiple worksheets. Retrieved June 16, 2020. https://kb. palisade.com/index.php?pg=kb.page \&id $=162$

Ridlehoover, J. (2004). Applying Monte Carlo simulation and risk analysis to the facility location problem. The Engineering Economist, 49(3), 237-252. https://doi.org/10.1080/00137910490498942

Sadyhova, E. (2020). Development of investment decision support tools based on the Simulation Decomposition method. Bachelor's thesis, Saint-Petersburg State University, Saint-Petersburg, Russia.

Vithayasrichareon, P. and Macgill, I.F. (2012). A Monte Carlo based decision-support tool for assessing generation portfolios in future carbon constrained electricity industries. Energy Policy, 41, 374392. 392. https://doi.org/10.1016/j.enpol.2011.10.060

Walker, S.G.B., Hipel K.W., and Inohara, T. (2012). Attitudes and preferences: Approaches to representing decision maker desires. Applied Mathematics and Computation, 218(12), 6637-6647. https://doi. org/10.1016/j.amc.2011.11.102 
Yeomans, J.S. (2008). Applications of simulation-optimization methods in environmental policy planning under uncertainty. Journal of Environmental Informatics, 12(2), 174-186. https://doi.org/10.3808 /jei.200800135
Zechman, E.M. and Ranjithan, S.R. (2004). An evolutionary algorithm to generate alternatives (EAGA) for engineering optimization problems. Engineering Optimization, 36(5), 539-553. https://doi. org/10.1080/03052150410001704863 